\title{
Texture-based Analysis of Fetal Organs in Fetal Growth Restriction
}

\author{
Aya Mutaz Zeidan ${ }^{1} \dagger^{*}$, Paula Ramirez Gilliland ${ }^{1} \dagger^{*}$, Ashay Patel $^{1}$, Zhanchong \\ $\mathrm{Ou}^{1}$, Dimitra Flouri ${ }^{1}$, Nada Mufti $^{1,2}$, Kasia Maksym ${ }^{2}$, Rosalind Aughwane ${ }^{2}$, \\ Sebastien Ourselin ${ }^{1}$, Anna David ${ }^{2}$, and Andrew Melbourne ${ }^{1}$ \\ ${ }^{1}$ School of Biomedical Engineering \& Imaging Sciences, King's College London, UK. \\ ${ }^{2}$ Institute for Women's Health, University College London, UK. \\ $\dagger$ Corresponding authors: \{aya.zeidan,paula.ramirez_gilliland\}@kcl.ac.uk \\ * The first two authors contributed equally.
}

\begin{abstract}
Fetal growth restriction (FGR) is common, affecting around $10 \%$ of all pregnancies. Growth restricted fetuses fail to achieve their genetically predetermined size and often weigh $<10$ th centile for gestation. However, even appropriately grown fetuses can be affected, with the diagnosis of FGR missed before birth. Babies with FGR have a higher rate of stillbirth, neonatal morbidity such as breathing problems, and neurodevelopmental delay. FGR is usually due to placental insufficiency leading to poor placental perfusion and fetal hypoxia. MRI is increasingly used to image the fetus and placenta. Here we explore the use of novel multi-compartment Intravoxel Incoherent Motion Model (IVIM)-based models for MRI fetal and placental analysis, to improve understanding of FGR and quantify abnormalities and biomarkers in fetal organs. In 12 normally grown and 12 FGR gestational-age matched pregnancies (Median $28^{+4}$ wks $\pm 3^{+3}$ wks) we acquired $\mathrm{T}_{2}$ relaxometry and diffusion MRI datasets. Decreased perfusion, pseudo-diffusion coefficient, and fetal blood $\mathrm{T}_{2}$ values in the placenta and fetal liver were significant features distinguishing between FGR and normal controls ( $\mathrm{p}$-value $<0.05$ ). This may be related to the preferential shunting of fetal blood away from the fetal liver to the fetal brain that occurs in placental insufficiency. These features were used to predict FGR diagnosis and gestational age at delivery using simple machine learning models. Texture analysis was explored to compare Haralick features between control and FGR fetuses, with the placenta and liver yielding the most significant differences between the groups. This project provides insights into the effect of FGR on fetal organs emphasizing the significant impact on the fetal liver and placenta, and the potential of an automated approach to diagnosis by leveraging simple machine learning models.
\end{abstract}

Keywords: FGR severity assessment - Multi-compartment models.

\section{Introduction}

The term Fetal Growth Restriction (FGR) is used to describe a fetus that has not reached their genetic growth potential, due to placental insufficiency causing 
inadequate supply of oxygen and nutrients [1]. FGR is a clinical diagnosis, defined by the Delphi consensus standardised definitions [2], and is divided into two different phenotypes, with onset either early (less than 32 weeks gestational age) or late in gestation. It is associated with high rates of stillbirth [3] and neonatal morbidity including increased rates of cerebral palsy, bronchopulmonary dysplasia, and cardiovascular disease long term [4]. There is currently no treatment for FGR, therefore clinicians must weigh the risks of prematurity against the risk of hypoxia and death in utero to determine the optimal delivery time. There are limited clinical tools to do this, so at present, clinicians follow national guidelines to make this decision [5].

MRI is increasingly used to image the placental circulation. The DECIDE multi-compartment model separates fetal and maternal flow characteristics of the placenta allowing measurement of the relative proportions of vascular spaces $[6,7]$. When applied in early-onset FGR, it identified reduced feto-placental blood oxygen saturation, where the degree of abnormality correlated with disease severity defined by ultrasound fetal and maternal arterial Doppler findings [8].

The motivation for this research was to compare MR derived parameters relating to perfusion and oxygenation within the placenta and three fetal organs (the brain, liver and lungs) between normally grown pregnancies and those complicated by early onset FGR, through multi-compartment models and texture analysis. Distinguishing features were then used to predict FGR diagnosis and gestational age (GA) at delivery via simple machine learning models.

\section{Methods}

\section{$2.1 \quad$ Data}

Patient MRI scans of voxel resolution $1.9 \times 1.9 \times 6 \mathrm{~mm}$ were acquired using the acquisition parameters from [6] (enabling both $\mathrm{T}_{2}$ relaxometry and diffusion MRI fitting), using a $1.5 \mathrm{~T}$ Siemens Avanto and performed under free-breathing. The dataset consisted of 12 early-onset FGR [2] ranging between $\left[24^{+2}, 33^{+6}\right]$ gestation weeks ${ }^{+ \text {days }}$, and 12 control pregnancies with MR data ranged between $\left[25^{+1}, 34^{+0}\right]$ GA interval, (Median $28^{+4}$ wks $\pm 3^{+3}$ wks) respectively. Specific details on subject inclusion criteria are available in [6]. The study was approved by the UK National Research Ethics Service and all participants gave written informed consent (REC reference 15/LO/1488).

There are biological mechanisms that may cause differences in the distribution of blood perfusion throughout the fetus in FGR. To investigate this, manual segmentation of the placenta, liver, lungs and brain was accomplished using itk-SNAP software. The resultant 3D mask files were used within the NiftyFit package for multi-parametric model-fitting [6], and to perform texture analysis.

\subsection{Model Fitting}

Model fitting techniques were applied to each organ segmentation over the averaged ROI signal and on a voxelwise scale, yielding quantitative metrics for 

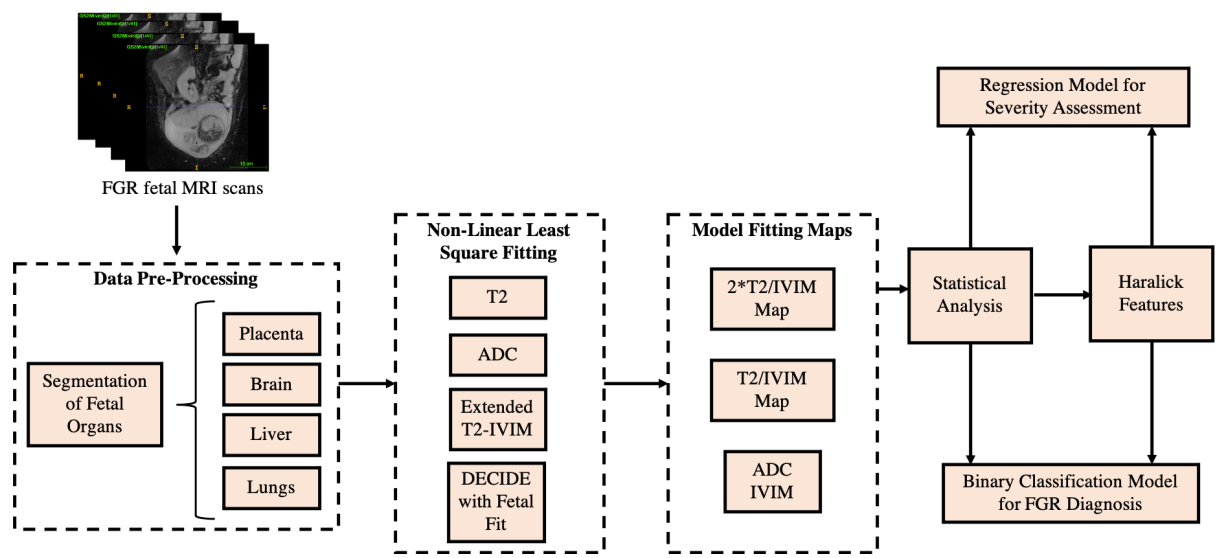

Fig. 1. Succeeding pre-processing of the data, model fitting techniques were applied to yield parameters describing various signals extracted from the placenta and fetal organs of interest. These parameters were then employed to perform texture analysis from multi-contrast MRI modelling. Results from the model fitting were used as inputs to the classifier and regressor to predict a diagnosis of FGR and the gestational age at delivery.

both approaches. Non-linear least squares were used to perform the fitting, with voxelwise fitting being initialised with the ROI parameter estimates - enhancing SNR. A range of models were explored, including simple $\mathrm{T}_{2}$ and Apparent Diffusion Coefficient (ADC) estimation, as well as more complex models based on Intravoxel Incoherent Motion Model (IVIM) [9] and DECIDE [6].

The IVIM model describes perfusion as a pseudodiffusion process (represented by a pseudodiffusion coefficient, $\mathrm{D}^{*}$ ), by characterising the collective motion of blood water molecules within the vessel network as a random walk. The IVIM model also incorporates "true" diffusion of water molecules (ADC), modelling the signal as

$$
S=S_{0}\left[f e^{-b \mathrm{D}^{*}}+(1-f) e^{-b \mathrm{ADC}}\right],
$$

where $f$ is the perfusion fraction (volume occupied by incoherently flowing blood in a given voxel), $b$ is the b-value, $S$ is the measured signal and $S_{0}$ the baseline signal, [10]. This can be extended to incorporate $T_{2}$ relaxometry as

$$
S=S_{0} e^{-t / \mathrm{T}_{2}}\left[f e^{-b \mathrm{D}^{*}}+(1-f) e^{-b \mathrm{ADC}}\right] .
$$

However, this model presents inherent limitations, as it assumes both vascular and tissue compartments (parametrised by pseudo-diffusion and true diffusion coefficients) have the same $\mathrm{T}_{2}$ value, leading to an overestimation of the pseudodiffusion volume fraction $f$ with increasing echo time $(t)$ [11].Thus, the analysis presented incorporates more complex models, accounting for varying blood and tissue $T_{2}$ values:

$$
S(\mathbf{b}, \mathbf{t})=S_{0}\left[f e^{-b \mathrm{D}^{*}} e^{-t / \mathrm{T}_{2 p}}+(1-f) e^{-b \mathrm{ADC}} e^{-t / \mathrm{T}_{2 t}}\right],
$$


with $f$ being the perfusion fraction, $\mathrm{T}_{2 p}$ and $\mathrm{T}_{2 t}$ being the transverse relaxation time for the pseudo-diffusion compartment (blood) and true diffusion compartment (tissue), respectively [11].

The DECIDE model [6] was also applied specifically to the placenta, which assumes three compartments with distinct diffusivity and relaxivity: fetal capillaries, trophoblast space and maternal blood pool. This model, given by Equation 4 , enables computation of novel placental biomarkers including maternal fetal blood volume ratio and fetal blood saturation.

$$
S(\mathbf{b}, \mathbf{t})=S_{0}\left[f e^{-b \mathrm{D}^{*}-t \mathrm{R}_{2}^{f b}}+(1-f) e^{-b \mathrm{ADC}}\left(\nu e^{-t \mathrm{R}_{2}^{m b}}+(1-\nu) e^{-t \mathrm{R}_{2}^{t s}}\right)\right] .
$$

Here, $\mathrm{R}_{2}^{f b}, \mathrm{R}_{2}^{m b}$ and $\mathrm{R}_{2}^{t s}$ represent the inverse of relaxation transverse relaxation times for fetal blood, maternal blood and trophoblast space, respectively; and $\nu$ is the maternal blood volume fraction.

\subsection{Texture Analysis}

The aim of texture analysis was to examine the spatial arrangement of intensities in the segmented organs. To achieve this, Haralick features were extracted from the grey level co-occurrence matrix to describe the overall image texture using measures encompassing energy, entropy, correlation, contrast, variance, and homogeneity [12].

These features were computed for each subject on all model fitting maps, as well as the original IVIM $\mathrm{T}_{2}$-weighted MRI scan, to yield interpretable texture descriptors $[12,13]$. The images were quantised into grey level bins of fixed equal width for between-subject texture feature value comparisons. Single-factor analysis of each feature was conducted between the FGR and control patients.

\subsection{Statistical Methods \& Feature Selection}

Statistical analysis was performed on the model fitting maps to identify the most significant parameters in differentiating between the control and FGR cohorts. A Shapiro-Wilk test was used to confirm normality of the results. T-tests were then carried out between the cohorts for all the model fitted parameters, Haralick features, and organ ratio parameters. Results with p-value less than 0.05 indicated statistically significant differences between the control and FGR group means.

\subsection{Machine Learning for FGR Diagnosis \& Severity Assessment}

Binary Classification Model A linear logistic regression model was trained to predict healthy or FGR using an $80 / 20 \%$ train-test split. The best regularisation parameters were derived by performing a grid search and 3-fold cross-validation, yielding a final model with regularisation strength of 0.001 and an L1 ratio of 0 , i.e. L2 regularisation. 
Regression Model A linear regression model was trained to predict the scanto-delivery interval, using clinical records data including the date of birth and the date on which the MRI scan was taken. The trained model had an elasticnet regularisation with an L1 ratio of 0.22 (i.e. a combination of L1 and L2 regularisation at a ratio of 0.22:0.78 respectively) and a regularisation strength of 0.0061 .

\section{Results}

\subsection{Model Fitting}

Figure 2 depicts examples of the parameter maps obtained from the model fitting techniques. The lower parameter map intensities in FGR compared to that in the controls is indicative of hypoperfusion and low oxygen saturation levels in these fetal organs. The $T_{2}$ maps display pronounced differences in the signal intensities of both cohorts. The most significant ROI and voxelwise parameters

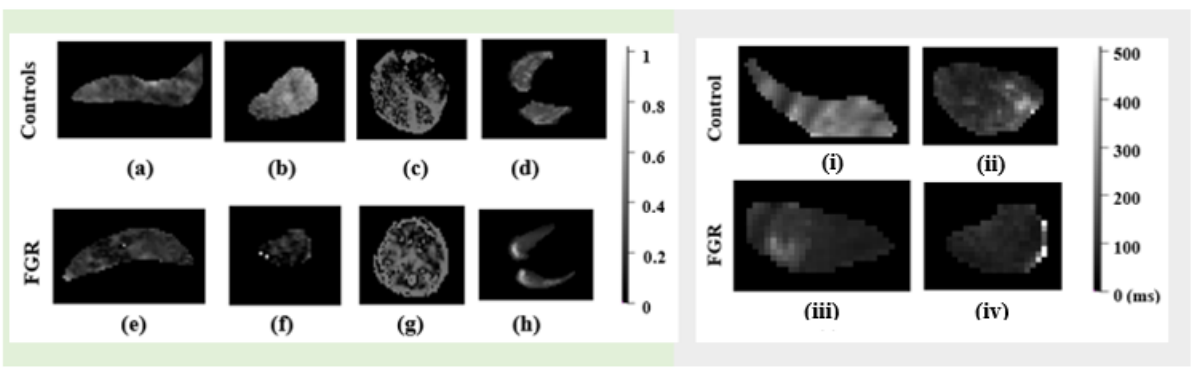

Fig. 2. Green shaded area plots: Perfusion fraction layer in the model fitting maps each taken from a single slice in the MRI scan. These correspond to $((\boldsymbol{a}),(\boldsymbol{e}))$ placenta, $((b),(f))$ liver, $((\boldsymbol{c}),(g))$ brain and $((d),(h))$ lungs. Grey shaded area plots: $\boldsymbol{T}_{2}$ maps for placenta ((i), (iii)), and liver ((ii), (iv)) from a single slice. In all cases top and bottom rows correspond to controls and FGR, respectively.

in identifying differences between controls and FGR fetuses were the perfusion fraction, $\mathrm{S}_{0}$, pseudo-diffusion coefficient $\left(\mathrm{D}^{*}\right)$, and $\mathrm{T}_{2}$ (as given in Tables 1 and 2 ). The placenta and liver were determined to be the most influential organs in diagnosing FGR.

The hierarchy of feature importances in Tables 1 and 2 specify that there were no significant differences detectable in the fetal brain and lungs between normal and FGR fetuses, especially compared to the placenta and liver, where differences were significant. This suggests that the brain and lungs may benefit from alternative analysis, focusing on certain cortical regions for the brain, and incorporating alternative imaging modalities for the lungs, as model fitting MRI analysis may not be the most appropriate technique for this fluid-filled organ. 


\begin{tabular}{|l||c|c|c|c|c|c|}
\hline \multicolumn{1}{|c||}{ Model Fitting Technique } & Parameter & $\begin{array}{c}\text { Average } \\
\text { Metric }\end{array}$ & $\begin{array}{c}\text { Pairwise Group } \\
\text { Comparison }\end{array}$ & Organ & T Statistic & P-Value \\
\hline \hline Standard IVIM & Perfusion Fraction & Mean & Control vs FGR & liver & 4.114582746 & 0.000494053 \\
\hline T2 Dependent IVIM & Perfusion Fraction & Mean & Control vs FGR & placenta & 2.885534725 & 0.008849743 \\
\hline DECIDE & Perfusion Fraction & Mean & Control vs FGR & placenta & 2.776531433 & 0.011308604 \\
\hline Standard IVIM & S0 & Min & Control vs FGR & placenta & 2.469368562 & 0.022196615 \\
\hline Standard IVIM & Perfusion Fraction & Mode & Control vs FGR & placenta & 2.440225199 & 0.023629376 \\
\hline ADC & Average S0 & Median & Control vs FGR & placenta & 1.985288036 & 0.060326406 \\
\hline Average T2 over ROI & Average T2 & Min & Control vs FGR & placenta & 1.928174375 & 0.067464017 \\
\hline T2 Dependent IVIM & T2 & Median & Control vs FGR & placenta & 1.926088314 & 0.067738281 \\
\hline Extended 2xT2 IVIM & Perfusion Fraction & Min & Control vs FGR & brain & 1.866272068 & 0.078382926 \\
\hline T2 Dependent IVIM & S0 & Mode & Control vs FGR & placenta & 1.815709446 & 0.083721797 \\
\hline
\end{tabular}

Table 1: Hierarchy of parameter feature importances of the ROI measurements.

\begin{tabular}{|l||c|c|c|c|c|c|}
\hline \multicolumn{1}{|c||}{ Model Fitting Technique } & Parameter & $\begin{array}{c}\text { Average } \\
\text { Metric }\end{array}$ & $\begin{array}{c}\text { Pairwise Group } \\
\text { Comparison }\end{array}$ & Organ & T Statistic & P-Value \\
\hline \hline Dependent IVIM & D $^{*}$ & Mean & Control vs FGR & placenta & -4.597300242 & 0.00015589 \\
\hline Extended 2xT2 Dependent IVIM & D $^{*}$ & Mean & Control vs FGR & placenta & -4.560436097 & 0.000170214 \\
\hline $\begin{array}{l}\text { DECIDE Model (Voxelwise } \\
\text { Measurements) }\end{array}$ & D $^{*}$ & Mean & Control vs FGR & placenta & -4.205788361 & 0.00039723 \\
\hline Extended 2xT2 Dependent IVIM & Perfusion Fraction & Min & Control vs FGR & placenta & 3.725183003 & 0.001250966 \\
\hline Extended 2xT2 Dependent IVIM & Perfusion Fraction & Mode & Control vs FGR & placenta & 3.725183003 & 0.001250966 \\
\hline Standard IVIM & Perfusion Fraction & Median & Control vs FGR & liver & 3.624757118 & 0.001587669 \\
\hline T2 Dependent IVIM & T2 & Min & Control vs FGR & placenta & 3.463092031 & 0.002326109 \\
\hline Extended 2xT2 Dependent IVIM & Perfusion Fraction & Median & Control vs FGR & placenta & 3.27041186 & 0.003653498 \\
\hline T2 Dependent IVIM & Perfusion Fraction & Min & Control vs FGR & placenta & 3.249455242 & 0.003836258 \\
\hline T2 Dependent IVIM & Perfusion Fraction & Mode & Control vs FGR & placenta & 3.249455242 & 0.003836258 \\
\hline
\end{tabular}

Table 2: Hierarchy of parameter feature importances of the voxelwise measurements.

\subsection{Texture Analysis}

Texture analysis was conducted on the most significant parameter maps for each organ, as determined by the t-tests. Results from the texture analysis were then concatenated by considering the mean and max of each Haralick feature.

Evaluation of the resulting Haralick features corroborated the degree of effect on the placenta in FGR, particularly using the Extended $\mathrm{T}_{2}$ IVIM map and its mean variance. The brain was the least significantly different organ in this analysis. The notches in the box plots delineate the extent of significant difference in the medians of the investigated features by representing the confidence interval of the metric. Greater mean variance in the signal from the Extended $\mathrm{T}_{2}$ IVIM model of the healthy cohort (refer to Figure 3(a)), is indicative of increased heterogeneity in FGR placentas. Max correlation of the liver perfusion fraction in the controls in Figure 3(d) reflects larger intensity differences compared to FGR. This is a significant feature to consider in the Standard IVIM model when studying the liver in FGR, especially given that the notches do not overlap between the cohorts. 


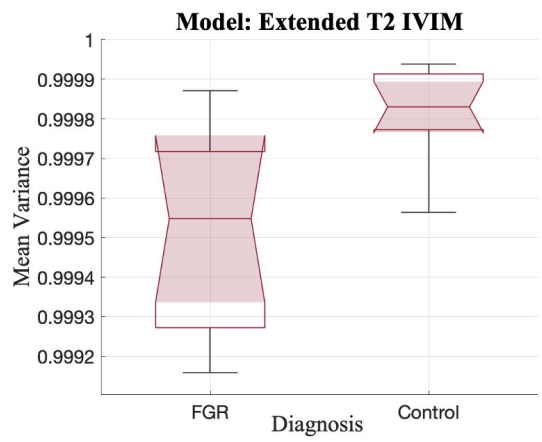

(a)

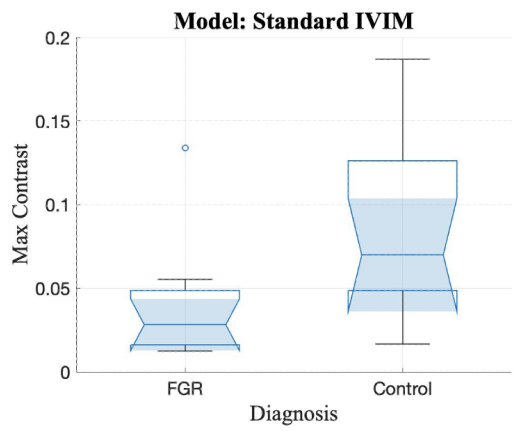

(c)

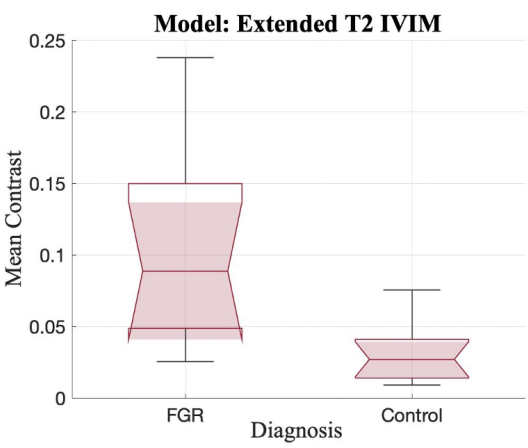

(b)

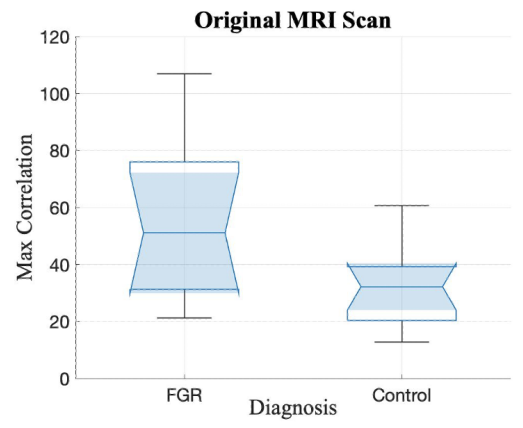

(d)

Fig. 3. Notched box plots of the most significant placental (pink) and liver (blue) Haralick features: $(\boldsymbol{a}, \boldsymbol{b})$ mean variance and contrast of the perfusion fraction in the Extended $T_{2}$ IVIM model, (c) max contrast of the Standard IVIM model, and (d) max correlation computed from the original scan.

\subsection{Machine Learning}

The classifier achieved a prediction accuracy of $100 \%$ in testing (refer to Table $3)$. Regarding the regressor, an RMSE of 0.02 weeks was achieved with the training data $(\mathrm{N}=18)$, in contrast with the RMSE of 3.06 weeks obtained on the test set $(\mathrm{N}=5)$. A generalisation error was evident, indicating a great degree of overfitting of the regression model on the training samples.

\section{Discussion}

In this study, we combined model fitting techniques, texture analysis from multicontrast MRI modelling, and machine learning, to facilitate multi-fetal organ analysis of FGR. This provided a more holistic approach to imaging this common pregnancy condition. Differences were observed, particularly in the placenta and fetal liver, emphasising the significant effect of FGR on these organs. 


\begin{tabular}{|c|c|c||c|c|c|}
\hline \multicolumn{3}{|c||}{ Cross Validation (N = 18) } & \multicolumn{3}{c|}{ Testing (N= 5) } \\
\hline \hline Accuracy & Sensitivity & Specificity & Accuracy & Sensitivity & Specificity \\
\hline $89 \%$ & $78 \%$ & $100 \%$ & $100 \%$ & $100 \%$ & $100 \%$ \\
\hline
\end{tabular}

Table 3: Classification Results.

Overall, the fitted model parameters reveal decreased perfusion fraction, $\mathrm{T}_{2}$, and $\mathrm{D}^{*}$ in the liver and placenta in FGR fetuses compared to the controls. These differences are indicative of a reduced oxygen saturation and perfusion within these organs, as well as abnormal capillary blood flow motion [8]. We did not observe significant differences in the properties of fetal brains and lungs between the FGR and control groups.

The machine learning analysis on these results supports the potential use of these parametric biomarkers in measuring FGR and providing an estimate of severity, including an indication of the likely GA at delivery. The classifier achieved $100 \%$ accuracy on testing data, indicating the model features are powerful indicators for FGR detection. But these results require prospective validation in a larger study population due to the small test group size in this proof-of-concept study. Moreover, a larger dataset would permit the transition into more complex prediction models in future research.

The RMSE of 3.06 weeks for the regressor's predictive performance encodes a large window in terms of fetal development. This method must therefore be refined before translation to a clinical environment. However, it may serve as a guide on condition severity. In practice though, this tool would also be used in conjunction with a wide range of information, including ultrasound data on fetal size, and maternal and fetal Doppler analysis of vascular resistance, which we have not included so far in this work.

The most influential Haralick features were extracted from the perfusion fraction measurements, particularly computed from the Extended $\mathrm{T}_{2}$ IVIM and Standard IVIM models. Another important parameter determined by the Haralick features was $\mathrm{T}_{2}$, attributed to its correlation with oxygen saturation (lower $\mathrm{T}_{2}$ reflects a lower oxygen saturation [14]).

The placenta was established as the organ with most significant textural differences between the FGR and control groups. Variance, contrast, entropy and energy in placental perfusion fraction maps were the most significant textural differences between FGR and controls. This may be related to differences in the presence of maternal and fetal vascular malformation $[15,16]$.

The second organ with greatest textural differences between both cohorts was the liver, particularly the pseudo-diffusion coefficient $\left(\mathrm{D}^{*}\right)$ maps (contrast, correlation, and energy), indicating spatial differences in the incoherent fetal capillary blood motion in this organ. This may indicate an abnormal blood motion in the liver compared to a healthy developing organ, affecting nutrient supply to this organ and may be related to the role of the ductus venosus in redistributing blood to the heart under the influence of increasing hypoxia [17]. 
Energy was heavily influenced by the number of grey levels and was, therefore, a significant feature for the placenta, lungs and brain, due to the presence of similar intensity voxels within local regions. Correlation was affected by the noise present in the image, which explains the notable correlation differences found in the liver, being the organ with the lowest SNR.

Analysis on parameter correlations indicated that as the perfusion fraction in the liver and placenta decreased, the more severely growth-restricted the FGR fetuses were. This corroborated our initial hypotheses for selecting the fetal liver and placenta as severely-affected organs in FGR, with SNR perhaps too low and variability too high to observe differences in the fetal brain and lung. Despite this, further work is needed to refine the analysis of the signals from these organs to better study the impact of FGR.

\section{Conclusion}

This study demonstrated the potential of MRI to improve holistic assessment of the fetus in FGR by assessing the vascular properties of highly-perfused fetal organs, via a multi-compartmental model fitting approach and texture analysis. The placenta and liver were prominent organs in identifying FGR fetuses, with key parametric features indicating a reduced perfusion, oxygenation and fetal capillary blood motion in these organs. Future work into multi-organ fetal analysis will extend these techniques to other placental complications in a larger-scale study.

Acknowledgements This research was supported by the Wellcome Trust (210182/Z/18/Z and Wellcome Trust/EPSRC NS/A000027/1) and the Radiological Research Trust. The funders had no direction in the study design, data collection, data analysis, manuscript preparation or publication decision.

\section{References}

1. Fiona Lyall, Stephen C Robson, and Judith N Bulmer. Spiral artery remodeling and trophoblast invasion in preeclampsia and fetal growth restriction: relationship to clinical outcome. Hypertension, 62(6):1046-1054, 2013.

2. S. J. Gordijn, I. M. Beune, B. Thilaganathan, A. Papageorghiou, A. A. Baschat, P. N. Baker, R. M. Silver, K. Wynia, and W. Ganzevoort. Consensus definition of fetal growth restriction: a Delphi procedure. Ultrasound in obstetrics 8 gynecology : the official journal of the International Society of Ultrasound in Obstetrics and Gynecology, 2016.

3. Jason Gardosi, Vichithranie Madurasinghe, Mandy Williams, Asad Malik, and André Francis. Maternal and fetal risk factors for stillbirth: Population based study. BMJ (Online), 346(7893), Feb 2013.

4. Marina Colella, Alice Frérot, Aline Rideau Batista Novais, and Olivier Baud. Neonatal and Long-Term Consequences of Fetal Growth Restriction. Current Pediatric Reviews, 14(4):212-218, Jul 2018. 
5. Green-top Guideline No. The investigation and management of the small-forgestational-age fetus. 2002.

6. Andrew Melbourne, Rosalind Aughwane, Magdalena Sokolska, David Owen, Giles Kendall, Dimitra Flouri, Alan Bainbridge, David Atkinson, Jan Deprest, Tom Vercauteren, et al. Separating fetal and maternal placenta circulations using multiparametric mri. Magnetic Resonance in Medicine, 81(1):350-361, 2019.

7. Sophie Couper, Alys Clark, John M D Thompson, Dimitra Flouri, Rosalind Aughwane, Anna L David, Andrew Melbourne, Ali Mirjalili, and Peter R Stone. The effects of maternal position, in late gestation pregnancy, on placental blood flow and oxygenation: An MRI study. The Journal of Physiology, 2020.

8. Rosalind Aughwane, Nada Mufti, Dimitra Flouri, Kasia Maksym, Rebecca Spencer, Magdalena Sokolska, Giles Kendall, David Atkinson, Alan Bainbridge, Jan Deprest, Tom Vercauteren, Sebastien Ourselin, Anna David, and Andrew Melbourne. MRI Measurement of Placental Perfusion and Oxygen Saturation in Early Onset Fetal Growth Restriction. BJOG: An International Journal of Obstetrics \&6 Gynaecology, pages 1471-0528.16387, Jun 2020.

9. Denis Le Bihan, Eric Breton, Denis Lallemand, Philippe Grenier, Emmanuel Cabanis, and Maurice Laval-Jeantet. Mr imaging of intravoxel incoherent motions: application to diffusion and perfusion in neurologic disorders. Radiology, 161(2):401407, 1986.

10. Denis Le Bihan. What can we see with ivim mri? Neuroimage, 187:56-67, 2019.

11. Neil P Jerome, JA d'Arcy, T Feiweier, DM Koh, MO Leach, DJ Collins, and MR Orton. Extended t2-ivim model for correction of te dependence of pseudodiffusion volume fraction in clinical diffusion-weighted magnetic resonance imaging. Physics in Medicine ES Biology, 61(24):N667, 2016.

12. Robert M Haralick, Karthikeyan Shanmugam, and Its' Hak Dinstein. Textural features for image classification. IEEE Transactions on systems, man, and cybernetics, 1(6):610-621, 1973.

13. Manish H Bharati, J Jay Liu, and John F MacGregor. Image texture analysis: methods and comparisons. Chemometrics and intelligent laboratory systems, 72(1):57-71, 2004.

14. Sharon Portnoy, Mark Osmond, Meng Yuan Zhu, Mike Seed, John G Sled, and Christopher K Macgowan. Relaxation properties of human umbilical cord blood at 1.5 tesla. Magnetic Resonance in Medicine, 77(4):1678-1690, 2017.

15. William Mifsud and Neil J. Sebire. Placental pathology in early-onset and lateonset fetal growth restriction. Fetal Diagnosis and Therapy, 36:117-128, 2014.

16. Graham J Burton, Andrew. W Woods, Eric Jauniaux, and John CP Kingdom. Rheological and Physiological Consequences of Conversion of the Maternal Spiral Arteries for Uteroplacental Blood Flow during Human Pregnancy. Placenta, 2009.

17. William Mifsud and Neil J Sebire. Placental pathology in early-onset and late-onset fetal growth restriction. Fetal diagnosis and therapy, 36(2):117-128, 2014. 